\title{
Left-right differences in motor thresholds after stimulation of the globus pallidus before pallidotomy
}

\author{
A Berić, D Sterio, M Dogali, P Kelly
}

\begin{abstract}
Left-right upper limb motor threshold differences were found after electrical stimulation of the globus pallidus administered as a neuroprotective measure to avoid lesioning of the internal capsule during stereotactic pallidotomy for treatment of Parkinson's disease. Left sided stimulation resulted in lower thresholds in right handed patients compared with left handed patients. These differences were significant in women, but no significant differences were found in men. In patients undergoing bilateral pallidotomy, the stimulation produced more significant left-right motor threshold differences. In the absence of known sex-related anatomical left-right corticospinal tract differences, the variability was the result of spinal excitability modulations most likely related to handedness.
\end{abstract}

(F Neurol Neurosurg Psychiatry 1997;63:159-162)

Keywords: motor thresholds; sex differences; pallidotomy; handedness; internal capsule

There are recognised differences between the functions of the left and right cerebral hemispheres. In humans, it is well established that language is related to the left hemisphere in right handed persons and, to a certain extent, in left handed persons. These differences can be identified by the WADA test. It has also been shown that the right hemisphere is involved in space orientation and most spatial tasks. In addition, it has been documented that left-right side differences exist in the motor tracts, especially in the corticospinal tract, with the left medullary pyramid being larger and decussating higher than on the right side. ${ }^{12}$ Furthermore, recently there have been reports of differences between excitability and thresholds of motor responses after non-invasive transcranial magnetic stimulation. ${ }^{34}$

We studied excitability of the corticospinal tract during posteroventral pallidotomy in patients with Parkinson's disease. ${ }^{5}$ The stimulating electrode was placed within the globus pallidus internus (GPi) next to the internal capsule (IC). The threshold of elicitation of the hand contraction was measured in patients who were awake and who did not receive anaesthetics just before placement of the radiofrequency lesion. We documented electrical thresholds for elicitation of contralateral upper limb muscle contraction after right or left GPi/IC stimulation, comparing results found in women with those found in men. Also, in patients undergoing staged bilateral pallidotomy, we were able to compare electrical thresholds after right and left stimulation in the same patient.

\section{Methods and materials}

Seventy three patients (30 women, 43 men) underwent unilateral posteroventral pallidotomy after providing their consent in accordance with the Institutional Review Board of the New York University Medical Center Hospital for Joint Diseases. Sixty one patients (26 women, 35 men) were right handed and 12 (four women, eight men) were left handed. They had predominantly unilateral symptoms with dominant bradykinesia, akinesia, and rigidity. All patients were evaluated by means of the core assessment program for intracerebral transplantation (CAPIT) protocol that incorporates the unified Parkinson's disease rating scale (UPDRS). ${ }^{67}$ Patients were evaluated in the morning after a 12 hour drug free period and about one hour after taking their medications when a typical best "on" state could be assessed. These assessments were repeated a day before pallidotomy. Patients were also scanned preoperatively with quantitative F-fluodeoxyglucose (FDG) PET. Results of these evaluations (CAPIT, UPDRS, and the PET) determined the side on which electrical stimulation and pallidotomy were performed. In addition, among the 73 patients, nine (five women, four men) underwent contralateral pallidotomy about one year after the unilateral procedure.

The procedure was performed under local anaesthesia. The pallidal target was defined with high resolution MRI. The LEKSELL frame was positioned with reference to the midpoint of the anterior commissure (AC)/ posterior commissure (PC) line. Acquired data were digitised on to a nine track tape and transferred to an independent workstation (CASS/Midco), which correlates fiducials in space, selects the most appropriate Shaltenbrand brain map, and performs preoperative planning of the target trajectories and target sites. The anatomical target generally lies between $19 \mathrm{~mm}$ and $21 \mathrm{~mm}$ lateral to the midline, 4-6 $\mathrm{mm}$ below the AC/PC line, $2-3 \mathrm{~mm}$ anterior to the mid-commissural point and 3-4 $\mathrm{mm}$ lateral and anterior to the internal capsule. The cranial opening was made with a $3.5 \mathrm{~mm}$ and in revised form 6

January 1997

Accepted 22 January 1997 
Table 1 Motor thresholds after left and right GPi/IC stimulation

\begin{tabular}{lllllll}
\hline Stimulation & Total & Right handed & Left handed & Women & Men & Bilateral \\
\hline Left GPi/IC & $4.9(1.4)^{\star}$ & $4.8(1.2)$ & $5.5(1.9)$ & $4.7(1.1)$ & $5.1(1.6)$ & $4.0(1.1)$ \\
Number & 36 & 29 & 7 & 16 & 20 & 9 \\
Right GPi/IC & $5.5(1.9)$ & $5.5(2.0)$ & $5.1(1.6)$ & $6.0(1.9)$ & $5.2(1.9)$ & $6.1(1.5)$ \\
Number & 37 & 32 & 5 & 14 & 23 & 9 \\
P< & 0.07 & 0.04 & 0.36 & 0.01 & 0.46 & 0.005 \\
Total (n) & 73 & 61 & 12 & 30 & 43 & 9 \\
\hline
\end{tabular}

*Mean (SD) in V.

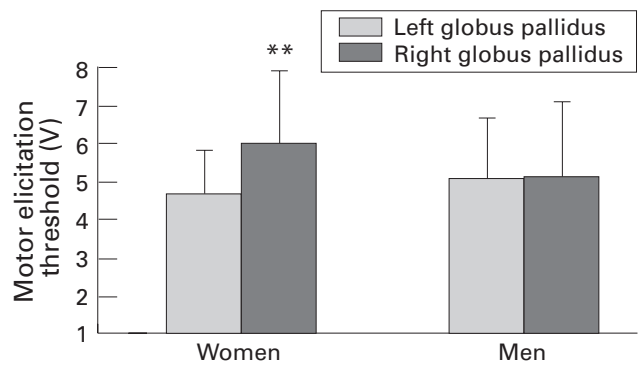

kin punch and $3 \mathrm{~mm}$ twist drill in the same plane as the $x$ coordinate (lateral to the midline), at an angle between $45^{\circ}$ and $50^{\circ}$ to the AC/PC plane.

Single cell microrecording was done to delineate the upper and lower borders of the GPi for precise localisation of the target site. ${ }^{8}$ Subsequently, a $1.1 \mathrm{~mm}$ diameter monopolar Radionics TM electrode with a $3 \mathrm{~mm}$ uninsulated tip was introduced to the target (macroelectrode). Electrical stimulation was carried out at the target site using an RF generator (Radionics Model RFG-3C). Biphasic, peaked pulses of $0.2 \mathrm{~ms}$ duration, $50 \mathrm{~Hz}$ frequency for three to four seconds, and intensity from $1.0 \mathrm{~V}$ to $10.0 \mathrm{~V}$ were applied. We measured the applied peak to peak current with an independent current probe (Tektronix A6302) displayed on a digital storage oscilloscope (Tektronix 2214). Intensity of stimulation was measured clinically, without EMG recording, by the method of limits. ${ }^{9}$ The intensity was increased and decreased three to five times until the motor response was repeatedly found at the same level of intensity (appearance threshold). The usual response consisted of wrist extension, finger flexion, thumb abduction or extension, and intrinsic hand muscle contraction. Muscle tone and voluntary muscle contractions of the patient's contralateral hand and arm were clinically examined repeatedly by the same investigator during the hour preceding electrical stimulation.

Statistical analysis was based on analysis of variance (ANOVA) to determine the significance between left and right side threshold differences. For patients who underwent bilateral pallidotomy, a paired two sample $t$ test was used to assess mean results. A P value $<0.05$ indicated that differences were significant. The statistical package Statistica (StatSoft, Inc, Tulsa, OK, USA) was used for the analysis.

\section{Results}

In the unilateral pallidotomies the surgery was performed on the side of the brain opposite to the side of the body associated with more severe symptoms of Parkinson's disease and dyskinesiae. There was no difference in severity of symptoms in women compared with men. Overall there was no difference in severity of symptoms on the right side of the body compared with the left.

The mean threshold at which contralateral hand contraction was found was $4.9 \mathrm{~V}$ after left $\mathrm{GPi} / \mathrm{IC}$ stimulation and $5.5 \mathrm{~V}$ after right $\mathrm{GPi} / \mathrm{IC}$ stimulation. When stimulation thresholds for the left and right side were analysed in only right handed patients, those differences became more pronounced (table 1). There
Figure 1 Difference between left and right IC hand motor elicitation thresholds in women compared with men. ${ }^{\star} P<0.01$.

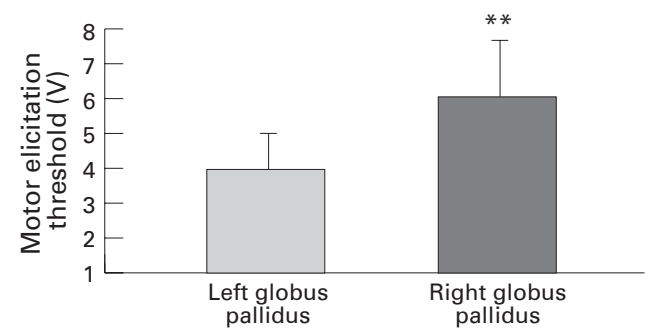

Figure 2 Mean hand motor thresholds after stimulation of left and right IC in patients who underwent bilateral pallidotomy. ${ }^{\star *} P<0.005$.

were no significant differences in the 12 left handed patients. Thresholds after left GPi/IC stimulation, however, were higher than those after right $\mathrm{GPi} / \mathrm{IC}$ stimulation, by contrast with what was found in right handed patients. Measurement of stimulation current showed the same general behaviour, which indirectly stimulations were within a relatively narrow range. The left GPi/IC stimulation showed a peak to peak current threshold of $14.0 \mathrm{~mA}$ whereas the right side stimulation threshold was $15.7 \mathrm{~mA}$.

When thresholds were analysed based on sex, there was a significant difference between left $(4.7 \mathrm{~V} / 12.7 \mathrm{~mA})$ and right $(6.0 \mathrm{~V} / 16.7 \mathrm{~mA})$ thresholds in women (fig 1). There were no significant differences, however, in those found in men $(5.1 \mathrm{~V} / 14.2 \mathrm{~mA}$ left $v 5.2 \mathrm{~V} / 15.9 \mathrm{~mA}$ right).

In all nine patients who underwent bilateral pallidotomy, stimulation threshold was lower on the left side than on the right and differences were highly significant. The threshold after left GPi/IC stimulation was $4.0 \mathrm{~V} / 10.2$ $\mathrm{mA} v 6.1 \mathrm{~V} / 17.0 \mathrm{~mA}$ after right GPi/IC stimulation (fig 2).

\section{Discussion}

We found differences between the thresholds that elicited hand contraction after stimulation of the left GPi/IC compared with the right. The thresholds were lower after left GPi/IC stimulation. Differences were even more prominent when results for right handed patients were analysed. Furthermore, analysis based on sex showed significant differences between left and right $\mathrm{GPi} / \mathrm{IC}$ stimulation thresholds in women but no differences in men. This was not due to the number of patients because more men than women underwent pallidotomy. Although the number of left handed patients was small for suggests that the impendances during our 
sound statistical comparison, threshold changes in these patients contrasted with those seen in the right handed patients. In patients who underwent bilateral pallidotomy, there were significant differences between left and right stimulation, further confirming the results associated with unilateral pallidotomy. There was no systematic error possible to explain these differences as about half of the patients first underwent right pallidotomy and a year later contralateral pallidotomy, whereas the other half had the opposite order of surgical sides.

The patients were relaxed during stimulation and the arm and hand muscles were continuously assessed for tone as well as voluntary and involuntary contractions. As none of the patients had muscle weakness, the differences in thresholds seem to be due to intrinsic differences between descending motor control of left and right muscles.

We think that the muscle contractions found were the result of direct spread of current to the corticospinal axons within the IC lying just medial and posterior to the stimulating electrode. Five independent lines of evidence show that the IC was a target of our stimulation: (1) We measured the motor thresholds at several points along the electrode trajectory. Initially, we took superior and anterior measurements within the globus pallidus externus and then proceeded through GPi down to the lesion target site, taking posterior and ventral measurements. Thresholds are always lowest at the deepest point along the trajectory, which is the most posterior and ventral point and therefore closest to the IC. (2) When several trajectories were used, the most medial had the lowest motor threshold, with the same distribution and appearance of facial and upper limb muscle contraction as occurred using more lateral trajectories. (3) Microelectrode recordings indicated that the posterior medial GPi area generated many lower limb responses. However, macroelectrode stimulation from the same site showed that the lowest motor thresholds were for the contraction of the upper limb and the face. Usually, in more lateral trajectories the microelectrode recorded responses from the hand where the macroelectrode stimulation would often lack any muscle contraction in either upper or lower limbs. (4) It is not surprising that pallidal stimulation does not result in muscle contraction, as the major pallidal output is inhibitory and directed towards the thalamus. (5) Intrinsic hand muscle EMG recordings during pallidal macrostimulation further supported IC stimulation. Using a single stimulus and employing voluntary facilitation of the target muscle, it was possible to obtain a reproducible EMG response from the abductor pollicis brevis muscle with latencies in the range of 16-18 ms and thresholds comparable with those elicited by trains of stimuli and assessed by palpation and visual inspection (unpublished). During complete relaxation, latencies would increase and responses would appear only with much higher intensities of stimulation. Described responses are reminiscent of the behaviour pattern of electrically induced, motor evoked responses (MEPs) with one exception: latencies of the transcranially elicited MEPs were generally 2-5 ms longer. ${ }^{10}$ In summary, it seems that macroelectrode stimulation of the pallidal target site results in direct spread of current to the neighbouring large myelinated fibres of the IC, leading to direct depolarisation of the spinal $\alpha$ motor neurons and consequent visible and palpable muscle contraction.

These findings support previous reports regarding left-right corticospinal tract differences noted after various stimulation methodologies, primarily in young, healthy volunteers. ${ }^{34}$ They also support overall recognition of anatomical differences found in the newborn $^{1}$ as well as in the general patient population studied at necropsy. ${ }^{21}$ Furthermore, our results obtained by stimulation of the corticospinal tract downstream, below the cortex, suggest that lower thresholds are due to differences in density, excitability, or synaptic efficacy of corticospinal to spinal motor neuron connections. This explanation supports the absence of cortical map hemispheric differences. ${ }^{12}$

Some morphological factors may also play a part in threshold differences. Morphological studies in the brainstem suggest a slightly larger left corticospinal tract. ${ }^{12}$ The left-right excitability differences were documented in both upper and lower limbs. ${ }^{3413-15}$ It also seems that right handed people show more asymmetry in brain function. This is suggested by more pronounced asymmetry in activation of the motor cortex during finger movement as measured by functional MRI. ${ }^{16}$ Other anatomical asymmetries are well documented, such as right-left temporal lobe differences. ${ }^{17}$ Also, in cognitive tasks of letter recall in half visual fields, right handed men showed asymmetry, whereas left handed men did not. ${ }^{18}$

This finding leads us to sex related differences in brain anatomy and function that may explain our main findings. Results indicating significant left-right threshold differences only in women and not in men suggest that there was no systematic error in the placement of the electrode in relation to the internal capsule, stereotactic frame, or the technique used to electrically stimulate these patients. However, such differences are difficult to explain. There is some indirect evidence that may help to understand these sex related differences. It has been claimed that lefthandedness is more prevalent in men, ${ }^{19}$ and women seem to function more quickly and skillfully with their right hand. ${ }^{20-23}$ Even in our small sample of patients with Parkinson's disease, we had more left handed men than women.

It is tempting to speculate that women may show more hand lateralisation favouring the right side. Assessment of some tactileperceptual functions supports this hypothesis. In a mixed right handed population, women scored better with their right hand, whereas men scored better with their left. ${ }^{24}$ Size of the corpus callosum and morphology of the sylvian fissure are different in men and women. ${ }^{25} 26$ There are also sex related differences in cogni- 
tive consequences after early hemispheric lesions. ${ }^{27}$ What seem to be needed are studies that will provide both perfomance testing and objective physiological correlates such as those provided by functional MRI or PET methodology. As this study did not include a comparison with healthy subjects, these results do not allow discussion regarding absolute leftright differences. They suggest, however, preservation of relative differences and basic organisation of the corticospinal direct pathways in patients with Parkinson's disease, even in relatively advanced stages.

1 Yakovlev PI, Rakic P. Patterns of decussation of bulbar pyramids and distribution of pyramidal tracts on two sides of the spinal cord. Trans Am Neurol Assoc 1966;91:366-7.

2 Kertesz A, Geschwind N. Patterns of pyramidal decussation and their relationship to handedness. Arch Neurol 1971;24: 326-32.

3 Macdonnell RAL, Shapiro BE, Chiappa KH, Helmers SL, Cros D, Day RJ, Shahani BT. Hemispheric threshold differences for motor evoked potentials produced by magnetic coil stimulation. Neurology 1991;41:1441-4.

4 Triggs WJ, Calvania R, Macdonnell RAL, Cros D, Chiappa KH. Physiological motor asymmetry in human handedness: evidence from transcranial magnetic stimulahandedness: evidence from tran

5 Dogali M, Fazzini E, Kolodny E, Eidelberg D, Sterio D, Devinsky O, Beric A. Stereotactic ventral pallidotomy for Devinsky O, Beric A. Stereotactic ventral pallidot
Parkinson's disease. Neurology 1995;45:753-61.

6 UPDRS Development Committee. Unified Parkinson's disease rating scale. In: Fahn S, Marsden CD, Clane DB Goldstein M, eds. Recent developments in Parkinson's disease. Vol 2. Florham Park, NJ: Macmillan, 1987:153-64.

7 CAPIT Committee. Core assessment program for intracerebral transplantation (CAPIT). Mov Disord 1992;7:2-13.

8 Sterio D, Berić A, Dogali M, Fazzini E, Alfaro G, Devinsky O. Neurophysiological properties of pallidal neurons in Parkinson's disease. Ann Neurol 1994;35:586-91.

9 Gescheider GA. Psychophysics: methods and theory. New York: Lawrence Erlbaum Associate Publishers, 1976:1177.

10 Berić A. Transcranial electrical and magnetic stimulation. In: Devinsky O, Beric A, Dogali M, eds. Electrical and magnetic stimulation of the brain and spinal cord, advances in neunetic stimulation of the brain and spinal cord, advances
rology. Vol 63. New York: Raven Press, 1993:29-42.
11 Nathan PW, Smith MC, Deacon P. The corticospinal tracts in man. Brain 1990;113:303-24.

12 Wilson SA, Thickbroom GW, Mastaglia FL. Transcranial magnetic stimulation mapping of the motor cortex in normal subjects. $\mathcal{F}$ Neurol Sci 1993;118:134-44.

13 Goode DJ, Glenn S, Manning AA Middleton JF. Lateral asymmetry of the Hoffmann reflex: relation to cortical laterality. F Neurol Neurosurg Psychiatry 1980;43:831-5.

14 Tan U. Relationship between hand skill and the excitability of motoneurones innervating the postural soleus muscle in human subjects. Int $\mathcal{F}$ Neurosci 1985;26:289-300.

15 Tan U. The H-reflex recovery curve from the wrist: lateralization of motoneuronal excitability in relation to handedness in normal subjects. Int $\mathcal{F}$ Neurosci 1989;48:271-84.

16 Kim S-G, Ashe J, Hendrich K, Ellermann JM, Merkle H, Ugurbil K, Georgopoulos AP. Functional magnetic resonance imaging of motor cortex: hemisph

17 Geschwind N, Levitsky W. Human brain: left-right asymmetries in temporal speech region. Science 1968;161:1867 .

18 Iaccino J. Asymmetrical processing of tachistoscopic inputs in undergraduates across sex, handedness, fieldside, and fixation instructions. Percept Mot Skills 1990;70:1203-13.

19 Geschwind N, Galaburda AM. Cerebral lateralization. Biological mechanisms, associations and pathology: a hypothesis and a program for research. Arch Neurol 1985;42:42859.

20 Tiffin J, Asher EJ. The Purdue pegboard: norms and studies of reliability and variability. $\mathcal{F}$ Appl Psychol 1948;32;234-47.

21 Gardner RA, Broman M. The Purdue pegboard: normative data on 1334 school children. f Clin Neuropsychol 1979;1:156-62

22 Mathiowetz V, Rogers SL, Dowe-Keval M, Donahoe L, Rennells C. The Purdue pegboard: norms for 14 to 19 year olds. Am f Occup Ther 1986;3:174-9.

23 Peters M, Servos P, Day R. Marked sex differences on a fine motor skill task disappear when finger size is used as covariate. 7 Appl Psychol 1990;75:87-90.

24 Genetta-Wadley A, Swirsky-Sacchetti T. Sex differences and handedness in hemispheric lateralization of tactile-spatial functions. Percept Mot Skills 1990;70:579-90.

25 Witelson SF. Hand and sex differences in the isthmus and genu of the human corpus callosum. Brain 1989;112:799835.

26 Witelson SF, Kigar DL. Sylvian fissure morphology and asymmetry in men and women: bilateral differences in relation to handedness in men. 7 Comp Neurol 1992;323: $326-40$

27 Strauss E, Wada J, Hunter M. Sex-related differences in cognitive consequences of early left-hemisphere lesions. $\mathcal{F}$ Clin Exp Neuropsychol 1992;14:738-48. 